\title{
Upper limits on density of dark matter in Solar system
}

\author{
I.B. Khriplovich ${ }^{1}$ \\ Budker Institute of Nuclear Physics \\ 11 Lavrentjev pr., 630090 Novosibirsk, Russia, \\ and Novosibirsk University \\ E.V. Pitjeva ${ }^{2}$ \\ Institute of Applied Astronomy \\ 10 Kutuzov Quay, 191187 St. Petersburg, Russia
}

\begin{abstract}
The analysis of the observational data for the secular perihelion precession of Mercury, Earth, and Mars, based on the EPM2004 ephemerides, results in new upper limits on density of dark matter in the Solar system.
\end{abstract}

\footnotetext{
${ }^{1}$ khriplovich@inp.nsk.su

2evp@quasar.ipa.nw.ru
} 
According to present observational data (see, for instance, [1]), the dark matter density in the region of the Milky Way Galaxy, where our Solar system is situated, is

$$
\rho_{\mathrm{dm}} \sim 0.5 \times 10^{-24} \mathrm{~g} / \mathrm{cm}^{3} .
$$

However, not so much is known about the local dark matter distribution inside our Solar system itself. A direct information on this distribution, even rather modest one as compared to the above number, is of certain interest.

Previous estimates of the dark matter density in the Solar system [2-4] resulted in the upper limits on the level of

$$
\rho_{\mathrm{dm}}<10^{-16} \mathrm{~g} / \mathrm{cm}^{3} .
$$

The bounds obtained in [2, 3] were based on the investigation of the possible effect of dark matter on the orbit of Uranus. On the other hand, the limit derived in [4] resulted from the analysis of the perihelion precession of the asteroid Icarus.

Here we present much stronger upper limits on the dark matter content in the Solar system following from analysis of the perihelion precession of Mercury, Earth, and Mars. Our limits are based on the precision EPM ephemerides constructed in [5] by simultaneous numerical integration of the equations of motion for the nine major planets, the Sun, and the Moon in the post-Newtonian approximation. Such subtle effects as the influence of 301 large asteroids and of the ring of small asteroids, as well as the solar oblateness, were included into the calculation. The EPM ephemerides resulted from a least squares adjustment to more than 317000 position observations (1913-2003) of different types, including radiometric and optical astrometric observations of spacecraft, planets, and their satellites.

The conclusions of the present paper are based on the possible corrections to the secular perihelion precession $\delta \phi$ (i.e. on the deviations of the results of theoretical calculations from the observational data) of three planets [6] obtained from about 250000 high-precision American and Russian ranging to planets and spacecraft (1961-2003), including in particular Viking-1,2, Pathfinder, Mars Global Syrveyor, and Odyssey. ${ }^{3}$ They are presented in Table 1.

\begin{tabular}{|c|c|c|c|}
\hline & Mercury & Earth & Mars \\
\hline " per century & $-0.0036 \pm 0.0050$ & $-0.0002 \pm 0.0004$ & $0.0001 \pm 0.0005$ \\
\hline$(\delta \phi / 2 \pi) \times 10^{11}$ & $-0.67 \pm 0.93$ & $-0.15 \pm 0.31$ & $0.14 \pm 0.73$ \\
\hline
\end{tabular}

Table 1. Corrections to secular perihelion precession of planets

\footnotetext{
${ }^{3}$ The corresponding correction from [6] to the perihelion precession of Venus is not used here, since it is much larger and less accurate. The reasons are as follows. On the one hand, Venus moves around the Sun more slowly than Mercury. On the other hand, for Venus there are no high-precision ranging, like those to martian landers or orbiting spacecraft, determining the accuracy of the corrections for Mars and Earth.
} 
Let us address now the possible influence of dark matter on the perihelion precession. To simplify our estimates, we will consider the dark matter as nonrelativistic dust with spherically symmetric density $\rho(r)$.

Then the correction $\delta F(r)$ to the gravitational force acting upon a planet with mass $m$ situated at a distance $r$ from the Sun is found easily by means of the Gauss theorem:

$$
F(r)=-k m \frac{\mu(r)}{r^{2}}
$$

where

$$
\mu(r)=4 \pi \int_{0}^{r} \rho\left(r_{1}\right) r_{1}^{2} d r_{1}
$$

is the total mass of dark matter inside a sphere of radius $r$, and $k$ is the Newton gravitational constant. The corresponding correction to the gravitational potential is

$$
\delta U(r)=k m \int_{0}^{r} d r_{2} \frac{\mu\left(r_{2}\right)}{r_{2}^{2}} .
$$

This correction shifts the perihelion of the planet orbit by angle

$$
\delta \phi=\frac{d}{d L} \frac{2 m}{L} \int_{0}^{\pi} d \phi r^{2} \delta U(r)
$$

per period [7; here $L$ is the planet angular momentum. With $\delta U(r)$ given by formula (4), and under the assumption (made also in $[2-4]$ ) that $\rho_{\mathrm{dm}}$ remains constant at the distances discussed, we arrive after some transformations at the result:

$$
\frac{\delta \phi}{2 \pi}=-\frac{3}{2} \frac{\mu(r)}{M_{\odot}} \sqrt{1-e^{2}}
$$

where $M_{\odot}$ is the mass of the Sun and $e$ is the eccentricity of the planet orbit. For Mercury, Earth, and Mars $e$ is small, about 0.21, 0.02, and 0.09, respectively, and will be neglected in the estimates below.

Now the results of [6] presented in Table 1 can be interpreted as upper limits on the mass of dark matter inside the orbits of corresponding planets. Using the data in the last line of Table 1, we formulate these upper limits as follows:

$$
\begin{gathered}
\mu(0.39 \mathrm{au})<6 \times 10^{-12} M_{\odot}, \\
\mu(1 \mathrm{au})<2 \times 10^{-12} M_{\odot} \\
\mu(1.52 \mathrm{au})<6 \times 10^{-12} M_{\odot}
\end{gathered}
$$

here we indicate in brackets the distance (in astronomical units, au) from the Sun of the corresponding planet, Mercury, Earth, and Mars, respectively.

At last, we convert these upper limits into the limits on the dark matter density. Then, the Mercury limit (7) results in the bound

$$
\rho_{\mathrm{dm}}<10^{-17} \mathrm{~g} / \mathrm{cm}^{3},
$$


somewhat better than (2).

The bounds following from the Earth and Mars limits, (8) and (9), are much stronger. They practically coincide and constitute

$$
\rho_{\mathrm{dm}}<3 \times 10^{-19} \mathrm{~g} / \mathrm{cm}^{3} .
$$

The result (11), though being far from the global (Galaxy) estimate (11), improves essentially the previous local (Solar system) limits (2).

We are grateful to A.E. Bondar, S.M. Kopeikin, A.A. Pomeransky, V.G. Serbo, and M.I. Vysotsky for the interest to the work, useful discussions, and pointing out two errors in the earlier versions of this note. The investigation was supported in part by the Russian Foundation for Basic Research through Grant No. 05-02-16627.

\section{References}

[1] G. Bertone and D. Merritt, Mod. Phys. Lett., A20 (2005) 1021; astro-ph/0504422.

[2] J.D. Anderson, E.L. Lau, A.H. Taylor, D.A. Dicus, D.C. Teplitz, and V.L. Teplitz, ApJ, $342(1989) 539$.

[3] J.D. Anderson, E.L. Lau, T.P. Krisher, D.A. Dicus, D.C. Rosenbaum, and V.L. Teplitz, ApJ, 448 (1995) 885; hep-ph/9503368.

[4] Ø. Grøn and H.H. Soleng, ApJ, 456 (1996) 445; astro-ph/9507051.

[5] E.V. Pitjeva, Solar System Research, 39 No 3 (2005) 176.

[6] E.V. Pitjeva, Astronomy Letters, 31 No 5 (2005) 340.

[7] L.D. Landau and E.M. Lifshitz, Mechanics, Pergamon Press, 1958. 